\title{
The role of intertrial interval in the learning of two simple avoidance tasks
}

\author{
ROBERT C. BOLLES and KELLY NELSON \\ University of Washington, Seattle, Washington 98105
}

\begin{abstract}
Rats were trained to avoid shock in either a jump-up or a one-way running situation with intertrial intervals of $20 \mathrm{~min}$ to $3 \mathrm{~h}$ for different groups. The rats with the shorter intervals learned quickly and performed well, but those with the longer intervals showed little learning. The performance of control rats suggests that these decrements occur because, with the longer intervals, the apparatus becomes a safe place.
\end{abstract}

The role of the intertrial interval (ITI) in avoidance learning has attracted relatively little systematic attention. However, an important study by Brush (1962) puts what is known about ITI effects fairly comfortably within the domain of two-factor theory. Brush found that the speed of avoidance learning in the shuttlebox increased with ITI up to approximately $5 \mathrm{~min}$ and then declined with longer ITIs. Brush's analysis was based upon a distinction between fear conditioned to the CS and fear conditioned to apparatus cues. The former is assumed to be relatively permanent, since response termination of the CS prevents the extinction of fear elicited by the CS. On the other hand, since apparatus cues persist throughout the ITI, fear conditioned to these cues should gradually dissipate in time. Learning is relatively poor with short ITIs because so much of the total fear is associated with apparatus cues that CS termination cannot provide a sufficient reduction in overall fear level to reinforce the response adequately. Performance deteriorates with long ITIs because there is so much dissipation of the apparatus component of fear that there is not enough total fear to adequately motivate the response. Thus, Brush suggests that the 5-min optimum ITI represents a compromise between inadequate reinforcement at shorter intervals and inadequate motivation at longer ones.

Although the basic features of Brush's analysis still seem defensible, there are some recent findings that pose some problems for it. One problem is that the fear conditioned to the CS appears to dissipate during the course of avoidance training so it is not clear how CS termination can continue being the source of reinforcement (e.g., Linden, 1969). A second difficulty with the conventional two-factor interpretation is that, as Denny (1971) has noted, the critical interval is probably not the time from the initiation of the trial but the time since the last shock. That is, fear conditioned to the apparatus must dissipate progressively over a series

Supported by research grant GB 40314 from the National Science Foundation. Requests for reprints should be addressed to the first author, Department of Psychology, University of Washington, Seattle, Washington 98195. of consecutive avoidance responses. There should, according to Brush's two-factor interpretation, be a loss of response strength over such a series of trials. The results often turn out quite differently; the first few consecutive avoidance responses are frequently a prelude to marked improvements in avoidance responding. Such considerations led Denny and his colleagues (Denny, 1971) to postulate the existence of a positive type of reinforcement mechanism. In any case, Denny's analysis encourages us not to attach so much importance to the ITI itself but to where the animal is and what it does between trials. The important consideration is said to be the development of relaxation during the ITI.

A third difficulty with the two-factor analysis is that it neglects the important consideration of what response is required of the animal. It seems likely that, in an avoidance task which is structured so that the appropriate response is the strongest one in the animal's repertoire, responding would be more dependent upon motivation and less upon reinforcement. A response that is initially weak, as in the shuttlebox task, might be initially more dependent upon reinforcement and less upon motivation. Thus, the optimum ITI should be expected to depend upon both the nature of the task and the stage of avoidance learning. There is, unfortunately, almost no data at the present time to test such predictions from the two-factor theory.

The purpose of the present experiment was to look at the effect of ITI in avoidance learning tasks other than the shuttlebox and to provide further investigation of long ITIs, $20 \mathrm{~min}$ and longer. The study shows that the important consideration is, in fact, not the length of the ITI but where the animal is during the ITI.

\section{EXPERIMENT I}

First we will look at the effects of long ITIs in a novel jump-out type of situation in which the animal can make a flight response from the shock area to a safe area, but the safe area is constructed so that the animal cannot remain there and so must necessarily spend the majority of the ITI in the dangerous part of the apparatus. 


\section{Method}

Subjects. The animals were 32 naive female rats of Wistar descent, approximately 90 days old.

Apparatus. The animals were trained in a box $23 \times 26 \times 18 \mathrm{~cm}$ high. Water and food in an external hopper were always available so that the animals could live in the apparatus for the long periods of time necessary under the long-ITI conditions. In the center of the ceiling was a wire cylindrically shaped cupola $5 \mathrm{~cm}$ in diam and $10 \mathrm{~cm}$ high. The cupola was attached loosely with a hinge on one side and a microswitch on the other so that the animals' entry into it could be recorded. Previous investigation had shown that rats quickly learned to escape shock by going into the cupola (Bolles, Riley, Cantor, \& Duncan, 1974) but the cupola is so confining that the animal cannot remain there more than a few minutes before it must drop back into the box. The floor of the box was constructed of $1-\mathrm{cm}$ stainless steel grids spaced $2.5 \mathrm{~cm}$ center to center. Shock was supplied at $.5 \mathrm{~mA}$ from a Grason-Stadler scrambler. A complex CS was used; it consisted of the experimenter entering the experimental room, immediately turning on a switch which sounded a 76-dB buzzer, turned on a $6-\mathrm{W}$ light bulb in the box, and started a 20 -sec clock which then initiated shock.

Procedure. The animals were randomly divided into four groups of eight and assigned to four ITI conditions which were $10 \mathrm{~min}, 20 \mathrm{~min}, 1 \mathrm{~h}$, and $3 \mathrm{~h}$. Since four cupola boxes were available, each group was run in two replications in a counterbalanced order. Animals were put into the boxes for $24 \mathrm{~h}$ prior to the beginning of training. All subjects were run 30 trials at the designated ITIs under conventional avoidance training conditions. If a response into the cupola occurred during the 20 -sec interstimulus interval, the shock was avoided. In the absence of an avoidance response, shock came on after $20 \mathrm{sec}$ and the subject was required to make the cupola response to terminate it.

\section{Results}

The number of avoidance responses over trial blocks for the four groups is shown in the left-hand part of Figure 1. It is apparent that learning proceeded quite rapidly with shorter ITIs. The median subject met a criterion of 9 out of 10 successful avoidances in less than 20 trials. We may presume that at the optimum ITI, some value less than $10 \mathrm{~min}$, this avoidance task is a very easy one for the rat to learn. However, there may be one difficulty in getting optimum performance with the cupola task. The rat may spend an appreciable period of time in the cupola, particularly on escape trials, and unless there is some device for forcing it back down into the box, it becomes technically impossible to run very short ITIs. It is apparent that, when the ITI was in the order of hours, the task became a much more difficult one, and perhaps impossible for the rat to learn. The differences among ITI conditions were highly reliable $[F(3,84)=16.9 ; p<.001]$. These results show the trend suggested by Brush's (1962) data, namely, a serious deterioration of avoidance learning with sufficiently long ITIs.

\section{Discussion}

The poor performance of subjects under the long-ITI conditions suggests that the ITI condition itself may be an important consideration, perhaps because the subject has a residual state of emotionality which gradually dissipates with time. Such an effect would lead us to regard the poor performance with the long ITI as a type of warm-up effect. Good performance may require that there be an appropriate emotional state which either persists from the preceding trial or has to be reinstated. In this connection, there is a tendency to think of fear conditioning as different from appetitive conditioning in one respect. In appetitive conditioning, we take for granted that there must be some prior state-hunger-before an animal will respond to a CS for food, but fear conditioning is assumed to be independent of the organism's prior state; it is always "prepared" to respond to a CS for shock. The present results suggest that this distinction may not be valid. Perhaps some prior condition of arousal or emotionality is required before a $\mathrm{CS}$ for shock will produce fear.

A second alternative is suggested by Brush's (1962) two-factor theory analysis. Perhaps some fear was elicited by the CS but because of the long ITI, apparatus fear had dissipated and the CS alone did not produce sufficient fear to motivate defensive behavior. This interpretation would be more convincing, however, if the task had been one which was inherently more difficult for the rat to learn. It is hard to believe that the rat needs much motivation in order to perform the natural defensive reaction that is required in the cupola apparatus. Another possibility is that the CS is a relatively unimportant consideration in the motivation of defense behavior, and that it serves primarily a discriminative function, telling the animal, in effect, when to respond. How to respond, we are suggesting, is primarily determined by the structure of the situation itself, i.e., by apparatus cues. What these results suggest in terms of this framework is that immediately upon being shocked and for some moments thereafter, apparatus cues are predictors of shock and produce an expectancy of shock. But after a period of time, perhaps 10 min or more, the shock situation is no longer a shock situation. The box becomes a safe place where relaxation and other activities occur. Because the box is a safe place at the start of the next trial, the rat remains there when the CS comes on. It has no reason to leave.

\section{EXPERIMENT II}

The purpose of Experiment II was to check the generality of these findings in a different rapidly learned avoidance situation, and to run some additional controls to help clarify the underlying mechanisms.

\section{Method}

Subjects. The animals were 44 naive female rats of Wistar descent approximately 90 days old.

Apparatus. The apparatus was a converted shuttlebox, each compartment of which was $38 \mathrm{~cm}$ long, $20 \mathrm{~cm}$ wide, and $18 \mathrm{~cm}$ high. One side was painted black and had a stainless steel grid floor with the same dimensions as that used in Experiment I. On the other side, the walls and floor were covered with white plastic inserts to facilitate discrimination between the two 
compartments. The CS was similar to that used in Experiment I; it consisted of the experimenter entering the room, tuming on a $20-\mathrm{sec}$ interstimulus timer and a $76-\mathrm{dB}$ buzzer, and opening the guillotine door separating the two compartments.

Procedure. Four experimental groups were randomly assigned eight animals each and were trained under conditions like those in Experiment 1 , with the ITI equal to $10 \mathrm{~min}, 20 \mathrm{~min}, 1 \mathrm{~h}$, and $3 \mathrm{~h}$ for the different groups. To make the conditions here as similar as possible to those of the cupola study, after the animal ran to the safe side of the apparatus, it was immediately forced by hand back through the door to the shock side where it remained during the ITI. Thus, these animals, like those in the cupola study, were required to spend the ITI in the dangerous part of the apparatus so that the four experimental groups constituted basically a replication of the cupola study.

In addition, three kinds of control groups were run, all with an ITI of $1 \mathrm{~h}$. One group $(\mathrm{N}=6)$ spent the ITI in the safe side of the apparatus. Another group $(\mathrm{N}=8)$ was removed from the safe compartment immediately after a response and spent the ITI in a neutral cage similar to its home cage to await placement in the shock side of the apparatus at the start of the next trial. A third control $(N=6)$ group was confined like the experimental groups to the shock side of the apparatus during the ITI, but to control for the potential effects of handling given the other controls at the initiation of a trial, the animal was picked upon from the shock side and returned to the shock side immediately prior to each trial.

\section{Results}

The percentage of avoidance responses over 10-trial blocks for experimental groups are shown in the right-hand side of Figure 1. It can be seen that the results closely parallel those of Experiment $I$. Performance deteriorated with the longer ITIs so that there was no indication of learning in the group with the 180 -min ITI, and the response was learned by all animals with the 10-min ITI, but the rate of learning was not as rapid as we would ordinarily expect in the one-way situation. Group differences were again reliable at the .001 level. Perhaps the 10-min ITI is already too long, and perhaps the confinement of the animal in the shock side of the apparatus during the ITI produces a decrement. These possibilities can be assessed by comparing experimental and control animals. The mean experimental animal with the 1-h ITI made 3.75 avoidances in 30 trials $(12.5 \%)$; the controls with the same ITI that spent the interval outside the shock compartment averaged $58 \%$ in the case of the group spending the ITI on the safe side and 53\% for the group spending the interval in the neutral apparatus. It would appear then that the debilitating effect on avoidance learning is primarily that the ITI is spent in the shock side of the apparatus. This treatment seems to preclude learning with long ITIs. That the superiority of the control groups is not due to their having been handled at the beginning of a trial is shown by the poor performance ( $1 \%$ avoidance) of the group spending the ITI in the shock side and handled just before each trial.

\section{Discussion}

It is apparent that the primary consideration in the detrimental effect of long ITI is not so much the length

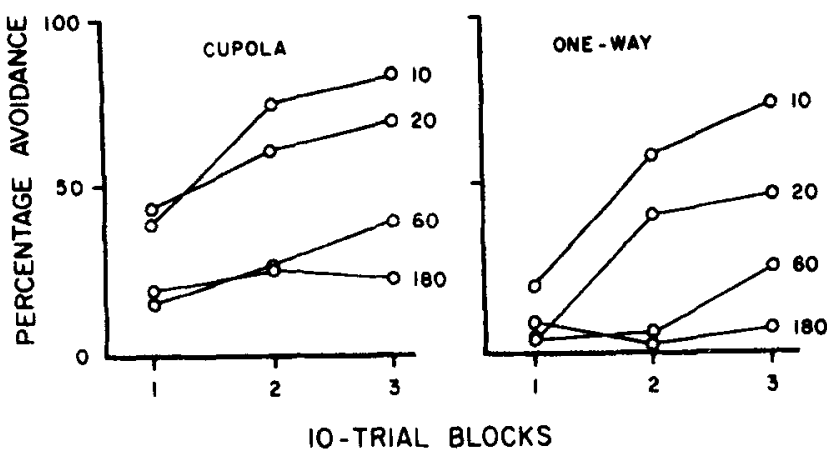

Figure 1. Percentage avoidance over trial blocks for rats in two situations as a function of intertrial interval.

of the ITI per se, but rather where the animal spends the ITI. Poor performance is found if the interval is spent in the dangerous part of the apparatus but not if it is spent in the safe part of the apparatus or in a neutral place.

Reynierse, Weisman, and Denny (1963) have previously reported decrements in one-way avoidance performance when animals are confined to the shock compartment during a large part of a 2-min ITI. Holloway and Wansley (1974) found similar decrements when their animals spent $15 \mathrm{sec}$ on a safe platform and up to $60 \mathrm{sec}$ on the grid floor, i.e., on the dangerous part of the apparatus. The present results confirm these findings and extend them by showing that when animals spend longer periods of time in the presence of danger cues, they show increasingly more serious decrements. A sufficiently long exposure to danger cues appears to make even the simplest avoidance tasks extremely difficult to learn.

This conclusion is consistent with Brush's (1962) two-factor interpretation of the effect of ITI on avoidance learning. The long ITIs spent in the dangerous part of the apparatus permits the fear conditioned to apparatus cues to dissipate. But in addition to this passive process, there is, we may suppose, a more active mechanism involved here. As Reynierse et al. describe it, when the rat spends an appreciable period of time in the shock situation following a shock, relaxation becomes conditioned to situational cues. This behavior then competes with the avoidance response. We would put it a little differently: the shock part of the apparatus becomes a safe place because the animal spends sufficient time there. Once in such a safe place, the animal stays there even when a CS predicting shock is presented.

\section{REFERENCES}

Bolles, R. C., Riley, A. L., Cantor, M. B., \& Duncan, P. M. The rat's failure to anticipate regulariy scheduled daily shock. Behavioral Biology, 1974, 11, 365-374.

Brush, F. R. The effects of intertrial interval on avoidance learning in the rat. Journal of Comparative and Physiological Psychology, 1962, 55, 888-892.

Denny, M. R. Relaxation theory and experiments. In F, R. Brush (Ed.), Aversive conditioning and learning. New York: 
Academic Press, 1971.

Holloway, F. A., \& Wansley, R. A. Motivational parameters in ethanol-induced state-dependent dissociation of avoidance learning. Physiological Psychology, 1974, 2, 71-74.

Linden, D. R. Attenuation and reestablishment of the CER by discriminated avoidance conditioning. Journal of Comparative and Physiological Psychology, 1969, 69, 573-578.
Reynierse, J. H., Weisman, R. G., \& Denny, M. R. Shock compartment confinement during the intertrial interval in avoidance learning. Psychological Record, 1963, 13,403-406.

(Received for publication July 22, 1974: revision received December 2,1974 .) 